\title{
Infrared temperature measurements on 3D objects with surface recession
}

\author{
by A. Fagnania,b , B. Helbera and O. Chazota \\ ${ }^{a}$ von Karman Institute for Fluid Dynamics, 1640, Rhode-Saint-Genèse, Belgium, andrea.fagnani@vki.ac.be \\ brije Universiteit Brussel, 1050, Brussel, Belgium
}

\begin{abstract}
The VKI Plasmatron is the world's largest inductively coupled plasma facility, which allows to reproduce on ground the high-enthalpy, chemically reacting boundary layer over a re-entry body. Test conditions are suitable for qualification of re-entry thermal protection systems and for material response characterization. Infrared thermography provides a valuable mean of non-intrusive surface temperature measurement on the test sample materials. However, infrared data are available in the form of two-dimensional images, while the test object usually has a three-dimensional shape. Moreover, the test material can undergo significant surface recession when exposed to the plasma flow. In this work, we propose a novel methodology to reconstruct three-dimensional temperature maps on a receding surface. First, an optical calibration technique is used to provide the necessary mapping parameters between the real world coordinates and the IR camera image pixels. Then, an auxiliary measurement of surface recession allows to determine the time evolution of the object shape. The surface temperature is hence reconstructed on the inferred time-varying geometry.
\end{abstract}

\section{Introduction}

The atmospheric entry of a spacecraft at hypersonic speed is a fascinating engineering challenge, as it involves a broad range of scientific and industrial capabilities. One crucial aspect is the design and the implementation of the Thermal Protection System (TPS), which is needed to protect the spacecraft and its payload from the severe thermal and mechanical loads encountered during the planetary entry. In this context, the use of ground test facilities for TPS characterization and qualification becomes imperative. During the last decades, growing attention has been paid to arc-jet and induction-type plasma wind tunnels, for their possibility to perform long duration, high-enthalpy tests [1]. Arc jets are usually preferred for qualification testing of large-scale samples at very high heat flux, thanks to their high power capabilities. On the other hand, Inductively Coupled Plasma (ICP) facilities, using electrodeless technology for the plasma discharge generation, ensure a superior flow purity and are usually more dedicated to the study of aerothermochemistry and gas-surface interaction phenomena [2]. TPS ground testing has a long experience at the VKI, where interest has been devoted to the material response characterization in terms of catalytic behaviour, oxidation processes and ablation phenomena. The work of Panerai [2] and Helber [3] extensively treated reusable and ablative heat shield testing, respectively.

Recently, in the course of the Design for Demise (D4D) strategy, promoted by the European Space Agency, the sound experience gained in TPS testing is being applied to study space debris materials. The D4D philosophy aims at reducing the risks associated with the ground impact of space debris through an appropriate design, by improving the break-up and demise mechanisms of any spacecraft component subjected to an uncontrolled atmospheric entry [4]. Plasma wind tunnel testing is herein crucial to develop and validate accurate models, suitable for predicting the thermal degradation process in re-entry conditions.

In this context, IR thermography is a valuable mean to perform temperature measurements in high-enthalpy facilities, because it is a non-intrusive optical technique, characterized by high sensitivity, low response time and it is fully two dimensional. However, while infrared data are available in the form of $2 \mathrm{D}$ images, the observed test object surface is often non-planar and the precise spatial reconstruction of the temperature maps becomes a critical task. Optical calibration techniques have already been introduced in IR thermography by Le Sant [5, 6, 7] and Cardone et al. [8]. These techniques adopt calibration targets or specifically designed control points on the investigated object, along with perspective projection models [9], to establish a relationship between the points of the observed surface and the image pixels, thus enabling the reconstruction of 3D temperature maps from the 2D thermal image. In addition, these procedures also allow to correct for the angular dependence of the material emissivity, since the angle of view with respect to the object surface normal can be easily computed [10].

In the VKI Plasmatron facility, however, the test material sample can undergo significant surface recession due to ablation phenomena which occur when interacting with the high-temperature, chemically reacting plasma flow. In the specific case of graphite, ablation of solid carbon is mainly due to heterogeneous and exothermic chemical reactions, such as oxidation by molecular and atomic oxygen, as well as nitridation reactions. For more detailed information about this, the reader may refer to [3]. Hence a traditional optical calibration procedure with a static target, in which the initial test object shape is known and kept constant for the entire test time, is no longer satisfactory. Instead, an improved procedure is needed to allow the dynamic tracking of the test object shape in time. In the present work, we introduce a novel experimental methodology which allows to accurately reconstruct the surface temperature maps onto ablating surfaces. 
The technique exploits high-speed camera imaging and edge detection algorithms to dynamically track the evolution of the test object shape in time. Under the assumption of axisymmetry, the target three-dimensional shape is then inferred from the observed two-dimensional lateral projection. Hence, the IR camera temperature map can be reconstructed on the time varying object shape.

Moreover, since measurements in high-enthalpy plasma flows may involve large temperature variations of the investigated object and chemical reactions with the flow, the change in optical properties of the emitting surface may severely affect the measurement. In particular, the object in-band emissivity, necessary for the correction of the IR thermograms to retrieve accurate temperature measurements, may change in a hardly predictable manner. In these conditions, comparative measurements between different techniques, such as pyrometry and radiometry, may help to retrieve more reliable estimates [11]. Here we also propose and implement computationally efficient algorithms for the in situ measurement and temperature-dependent correction of the in-band emissivity, by comparison with a simultaneous ratio pyrometry.

The proposed techniques aims at improving the material response characterization in plasma facilities, allowing to retrieve multidimensional temperature information more precisely and allowing enhanced comparison with material response simulation codes.

\section{Experimental facility and measurement techniques}

\subsection{VKI Plasmatron facility description and operating conditions}

The VKI Plasmatron is the world's largest ICP facility, equipped with a $160 \mathrm{~mm}$ diameter torch powered by a highfrequency, high-power, high-voltage $(400 \mathrm{kHz}, 1.2 \mathrm{MW}, 2 \mathrm{kV})$ electric generator. The flow regime is typically subsonic, although sonic and supersonic flow conditions can also be achieved by means of a nozzle configuration (not performed in this work). As the gas is heated by electromagnetic induction, the main advantage of ICP torches with respect to arc-jet heaters is the high purity of the plasma flows, due to the absence of chemical pollution associated to the erosion of the electrodes. In addition to this primary advantage, the ICP wind tunnel can work as a good plasma source, generating a quasistationary and Local-Thermochemical-Equilibrium (LTE) plasma in the downstream region of the heater, when the flow in the test section is low subsonic and the pressure remains sufficiently high. A schematic of the facility, representing the test chamber, torch, electric generator and cooling system, can be found in Fig. 1. A $30 \mathrm{~mm}$ diameter hemispherical probe, holding a $14 \mathrm{~mm}$ copper calorimeter in the center of the front face, is used to calibrate the plasma flow conditions. The Cold Wall $(\mathrm{CW})\left(T_{\mathrm{cw}} \cong 350 \mathrm{~K}\right)$ stagnation point heat flux $\left(\dot{q}_{\mathrm{cw}}\right)$ is determined by the cooling water mass flow, controlled by a calibrated rotameter, and the inlet/outlet temperature difference, measured by two type-E thermocouples. Both the probe and calorimeter have copper surfaces which are highly catalytic to nitrogen and oxygen atoms recombination. A water-cooled Pitot probe is used to perform dynamic pressure $\left(p_{\text {dyn }}\right)$ measurements. The pressure line is connected to a Validyne variable reluctance pressure transducer and a voltage demodulator amplifies the signal. Validyne and amplifier are calibrated prior to the test by means of a Betz water manometer. The probes are mounted at $380 \mathrm{~mm}$ distance from the ICP torch exit and the placement ensures that the axis of the probe and the axis of the torch itself coincide. An absolute pressure transducer (Memberanovac DM 12, Leybold Vacuum) measures the static pressure $\left(p_{\mathrm{s}}\right)$ in the Plasmatron test chamber. As the plasma jet is low subsonic $(M a \approx 0.1)$, the static pressure can be assumed constant in the whole test chamber. This assumption is confirmed a posteriori by the numerical simulations of the plasma jet. The gas mass flow rate $\left(\dot{m}_{\text {gas }}\right)$ supplied to the torch is monitored through a calibrated gas rotameter, while the Plasmatron control system allows to record the electric power $\left(P_{\mathrm{el}}\right)$ supplied to the induction coil.

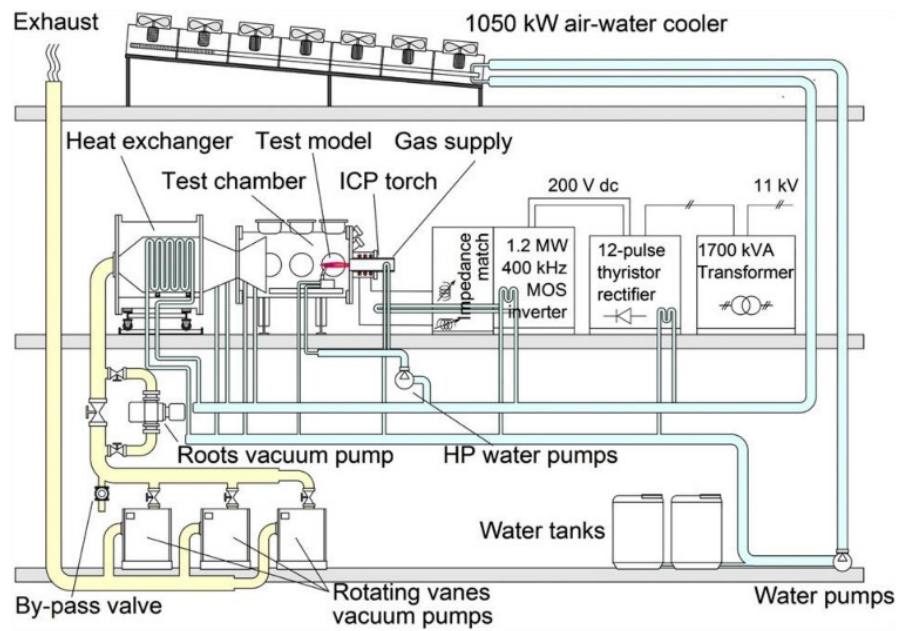

Fig.1. Schematic of the VKI Plasmatron facility, highlighting the 1.2 MW electric generator, the ICP torch, the test chamber and the cooling system. 


\subsection{Experimental methods}

\subsubsection{Experimental set-up}

A schematic of the experimental set-up used in the context of this work can be found in Fig. 2 (left). The test sample is a $30 \mathrm{~mm}$ diameter hemisphere-cylinder made of graphite and suitably insulated, by means of an alumina-silica disc, from a graphite support and mounted on a cooled probe holder, as shown in Fig. 2 (right). Heat flux and Pitot probes are used to characterize the plasma flow conditions, by measuring the cold-wall heat flux $\dot{q}_{\mathrm{cw}}$ and the jet dynamic pressure $p_{\text {dyn. }}$. A two-color pyrometer is pointed at the stagnation region, in the front part of the test sample. An infrared thermocamera is used to asses the sample surface temperature distribution and optical access to the Plasmatron test chamber is offered through a KRS-5 window. A high-speed camera is mounted laterally to record the time evolution of the test sample shape. All the instruments are synchronized by means of a trigger unit. Further details on each instrument are provided in the following sections.
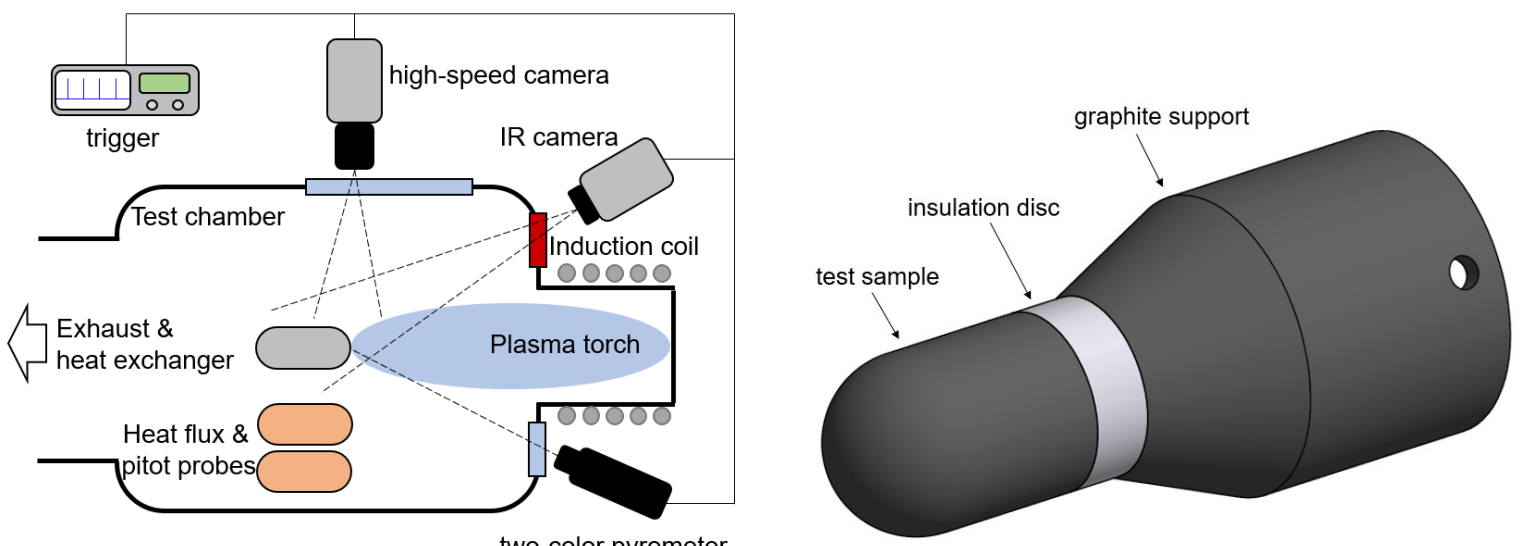

Fig. 2. Schematic of the experimental set-up, highlighting the IR camera, two-colour pyrometer, high-speed camera and trigger unit for synchronization (left). $3 D$ view of the test probe, featuring the graphite test sample, the alumina-silica insulation disc and the graphite support (right).

\subsubsection{Surface pyrometry}

The main drawback of optical temperature measurements is related to the uncertainty in the value of the surface emissivity. Two-colour pyrometry provides an emissivity-independent temperature measurement by recording the radiance at two different wavelengths. Assuming a grey-body behaviour of the surface (i.e., constant emissivity over the sensitive wavebands of the instrument), one manages to obtain a temperature measurement independent of emissivity. From Plank's law, the ratio of the spectral radiance at two different wavelengths, $\lambda_{1}$ and $\lambda_{2}$, reads

$$
\frac{I\left(\lambda_{1}, T\right)}{I\left(\lambda_{2}, T\right)}=\frac{\varepsilon_{\lambda_{1}}}{\varepsilon_{\lambda_{2}}}\left(\frac{\lambda_{1}}{\lambda_{2}}\right)^{5} e^{\frac{\mathrm{hc}}{k_{B} \lambda T}\left(\frac{1}{\lambda_{2}}-\frac{1}{\lambda_{1}}\right)}
$$

Hence, by considering $\varepsilon_{\lambda_{1}}=\varepsilon_{\lambda_{2}}$ and knowing $\lambda_{1}$ and $\lambda_{2}$, this ratio only depends on temperature. We used the two-colour Raytek Marathon Series MRS1-C pyrometer (Raytek Corporation, Santa Cruz, California, USA), employing a wide (0.751.1 mum) an a narrow (0.95-1.1 mum) spectral bands, at $10 \mathrm{~Hz}$ acquisition rate. The pyrometer has been calibrated for the range $1300-3300 \mathrm{~K}$, with a reference blackbody source. Optical access to the test chamber is offered through a $1 \mathrm{~cm}$ thick quartz window, placed at $\sim 1 \mathrm{~m}$ distance from the test sample, with an orientation of 35 degrees with respect to the jet axis. The pyrometer is pointed at the stagnation point and the measurement spot on the material sample has an estimated elliptical shape with dimensions $5.6 \mathrm{~mm}$ major axis, $4.6 \mathrm{~mm}$ minor axis. The measurement uncertainty is estimated at $\pm 1.5 \%$.

\subsubsection{Infrared thermography}

The infrared thermocamera FLIR A6750sc (FLIR Systems, Wilsonville, Oregon, USA) is used to assess the temperature distribution over the test sample material. It is equipped with an Indium Antimonide (InSb) detector, with a sensitive spectral range within $1-5 \mu \mathrm{m}$ and a resolution of $640 \times 512$ pixels. The camera is equipped with a $50 \mathrm{~mm}$ lens with 
a reduced spectral range from $3 \mu \mathrm{m}$ to $5 \mu \mathrm{m}$ and an intensity filter, which allows a measurement range up to $3300 \mathrm{~K}$. The camera is placed at $\sim 1 \mathrm{~m}$ distance from the test sample, with an inclination of 47 degrees with respect to the jet axis and optical access to the Plasmatron chamber is provided through a $1 \mathrm{~cm}$ thick KRS-5 window. The field of view at the test sample distance is approximatively $192 \times 154 \mathrm{~mm}^{2}$, resulting in a spatial resolution of $0.3 \mathrm{~mm} / \mathrm{pixel}$ on a planar target. The camera has been calibrated in intensity with a reference blackbody source between $800 \mathrm{~K}$ and $3300 \mathrm{~K}$.

\subsubsection{IR theory}

According to Plank's law, the spectral radiance $I(\lambda, T)$, emitted at a wavelength $\lambda$ by a body at temperature $T$ is

$$
I(\lambda, T)=\varepsilon(\lambda, \vartheta, T) \frac{2 \mathrm{hc} c^{2}}{\lambda^{5} e^{\frac{h c}{k_{B} \lambda T^{-1}}}}
$$

where $\varepsilon(\lambda, \vartheta, T)$ is the spectral emissivity, $\vartheta$ is the viewing angle, while $\mathrm{h}, \mathrm{c}$ and $\mathrm{k}_{\mathrm{B}}$ are the Plank's constant, the speed of light and the Boltzmann constant, respectively. A radiometer measures the in-band radiance $I_{\text {meas }}$, emitted and reflected by an object surface, in a certain waveband $\Delta \lambda$, characterized by the in-band emissivity $\varepsilon_{\Delta \lambda}$, and it can be expressed as [8]

$$
I_{\text {meas }}=\tau \varepsilon_{\Delta \lambda}(\vartheta, T) I_{\mathrm{obj}}^{\mathrm{BB}}+\tau\left[1-\varepsilon_{\Delta \lambda}(\vartheta, T)\right] I_{\mathrm{amb}}^{\mathrm{BB}}+(1-\tau) I_{\mathrm{atm}}^{\mathrm{BB}}
$$

where $\tau$ is the transmissivity of the optical path between the sample surface and the camera sensor, $I_{\mathrm{obj}}^{\mathrm{BB}}$ is the black-body radiation intensity corresponding to the object temperature $T_{\mathbf{o b j}}, I_{\mathrm{amb}}^{\mathrm{BB}}$ is the black-body radiation intensity corresponding to the equivalent ambient temperature $T_{\mathrm{amb}}$ and $I_{\mathrm{atm}}^{\mathrm{BB}}$ is the black-body radiation intensity emitted by the atmosphere along the optical path, corresponding to the equivalent atmosphere temperature $T_{\mathrm{atm}}$. Assuming the IR camera output signal $U_{\text {meas }}$ to be proportional to the received radiation intensity $I$, inverting the previous equation, one obtains

$$
U_{\mathrm{obj}}^{\mathrm{BB}}=\frac{1}{\tau \varepsilon_{\Delta \lambda}} U_{\text {meas }}-\frac{1-\varepsilon_{\Delta \lambda}}{\varepsilon_{\Delta \lambda}} U_{\mathrm{amb}}^{\mathrm{BB}}-\frac{1-\tau}{\tau \varepsilon_{\Delta \lambda}} U_{\mathrm{atm}}
$$

After the camera is calibrated under well controlled conditions, the signal intensity $U_{\mathrm{obj}}^{\mathrm{BB}}$ can be related to the object temperature $T_{\mathrm{obj}}$, typically through the non-linear relation $T_{\mathrm{obj}}=\mathcal{F}\left(U_{\mathrm{obj}}^{\mathrm{BB}}\right)$, once the in-band emissivity $\varepsilon_{\Delta \lambda}$, the optical path transmissivity $\tau$, the equivalent ambient temperature $T_{\mathrm{amb}}$ and atmosphere temperature $T_{a t m}$ are known. For typical applications in the VKI Plasmatron facility, the ambient radiation intensity can be reasonably neglected. Owing to the very high surface temperature $\left(T_{\mathrm{obj}}>1000 \mathrm{~K}\right)$ and emissivity $\left(\varepsilon_{\Delta \lambda}>0.7\right)$ of the test sample, in fact, we have $I_{\mathrm{amb}}^{\mathrm{BB}}<<I_{\mathrm{obj}}^{\mathrm{BB}}$ and $\left(1-\varepsilon_{\Delta \lambda}\right) / \varepsilon_{\Delta \lambda}<<1$, which both contribute to making the second term in eq. 4 negligible. However, the plasma flow is a strong emitter of electromagnetic radiation, and particular attention must be paid to evaluating the transmissivity $\tau$ and the radiation intensity $I_{\mathrm{atm}}$ along the optical path. Current efforts are being undertaken at VKI to quantify in detail the effect of the plasma emission on the radiometric measurements. In particular, the temperature distribution along the line-of-sight of the instrument is extracted from numerical simulations of the plasma flow field, accomplished with an in-house code $[12,13]$. The radiative transfer equation is then solved along the slab and the plasma emission is compared to the surface radiance. Preliminary results show that the plasma transmissivity in the wavelength range of interest for IR thermography is usually very close to one and the radiation intensity $I_{\mathrm{atm}}^{\mathrm{BB}}$ is also negligible compared to $I_{\mathrm{obj}}^{\mathrm{BB}}$, if the object surface temperature is large enough (typically if $T_{\mathrm{obj}}>1000 \mathrm{~K}$ ). It must be noted that the KRS-5 window, instead, has a nonnegligible attenuation of the radiation signal $\left(\tau_{K R S-5} \cong 0.7\right)$, which, however, is already accounted for in the calibration of the instrument. As a result, for our applications, we can reasonably assume $\tau \cong 1$ and neglect also the third term at the right end side of eq. 4 , which finally reduces to

$$
U_{\mathrm{obj}}^{\mathrm{BB}} \cong \frac{1}{\varepsilon_{\Delta \lambda}} U_{\text {meas }}
$$




\subsubsection{In situ measurement and temperature dependent correction for the in-band emissivity}

To correctly determine the surface temperature of the test object, it is still necessary to correct the recorded IR signal intensity $U_{\text {meas }}$ for the in-band emissivity $\varepsilon_{\Delta \lambda}$, before applying the intensity calibration $T_{\mathrm{obj}}=\mathcal{F}\left(U_{\mathrm{obj}}^{\mathrm{BB}}\right)$ [8]. Using tabulated values for the materials of interest usually implies unsatisfactory results and large uncertainty on the measured temperature. The material surface, in fact, may span a very large temperature range, from room temperature up to 3000 $\mathrm{K}$. It is then generally wrong to assume a constant value for $\varepsilon_{\Delta \lambda}$. Moreover, the test material can undergo heterogeneous reactions when exposed to the high-temperature, chemically reacting plasma flow. These, in turn, may alter the surface chemical composition, thus changing its optical properties in an unpredictable manner [2].

A specific procedure, comparing the emissivity-independent temperature measurement, provided by the two-colour pyrometer, with the calibrated IR camera signal was developed to provide a time-resolved and temperature-dependent in situ measurement of the in-band emissivity. Once this coefficient is determined, the IR camera frame intensity can be corrected accordingly, hence providing an accurate temperature measurement. The major assumption in this case is that the angular dependence of the in-band emissivity is neglected, and the value retrieved from the relative orientation between the two-colour pyrometer and IR camera is used for each point on the test sample surface. For materials of interest for the Plasmatron facility, however, the angular dependence of the spectral emissivity coefficient is typically negligible for viewing angles less than ${ }^{\circ} 50$ [10], which is the case for the test sample surface orientation with respect to the IR camera.

Moreover, iterative procedures must be established either for the determination of $\varepsilon_{\Delta \lambda}$ and for the correction of the measured signal intensity, due to the non-linear behaviour of both $\mathcal{F}\left(U_{\mathrm{obj}}^{\mathrm{BB}}\right)$ and $\varepsilon_{\Delta \lambda}(T)$. The procedure to determine $\varepsilon_{\Delta \lambda}(T)$ is generally very fast, since it has to act on a single IR camera frame pixel, or, at most, on an averaged frame area corresponding to the measurement location of the two-colour pyrometer. On the other hand, the procedure to correct the frame intensity for the in-band emissivity is computationally expensive, since it has to act on all the frame pixels associated to the test sample (typically in the order of $10^{5}$ ), for all the frames of the IR video sequence (typically in the order of $5 \cdot 10^{3}$ ). An efficient Newton method was implemented for this purpose, able to perform a full frame emissivity correction in around $0.5 \mathrm{~s}$. A schematic of these procedures is provided in Fig. 3.

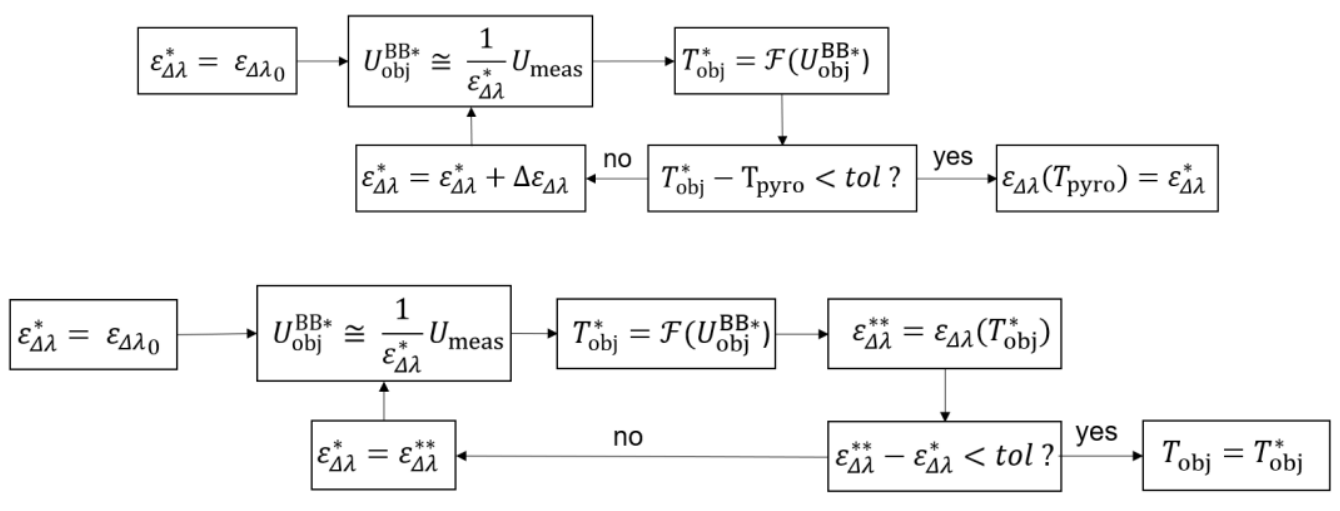

Fig. 3. Schematic of the in-band emissivity measurement (top) and temperature dependent correction (bottom) procedures.

\subsubsection{Image recognition applied to infrared thermography}

After proper intensity calibration and correction for emissivity, IR thermography provides a two-dimensional projection of an object temperature map. To exploit the multidimensional temperature information for quantitative measurements, however, it is required to establish the correspondence between the points of the observed surface and the pixels of the thermal image. This procedure requires dedicated processing techniques if the object has a non-planar geometry [8]. 


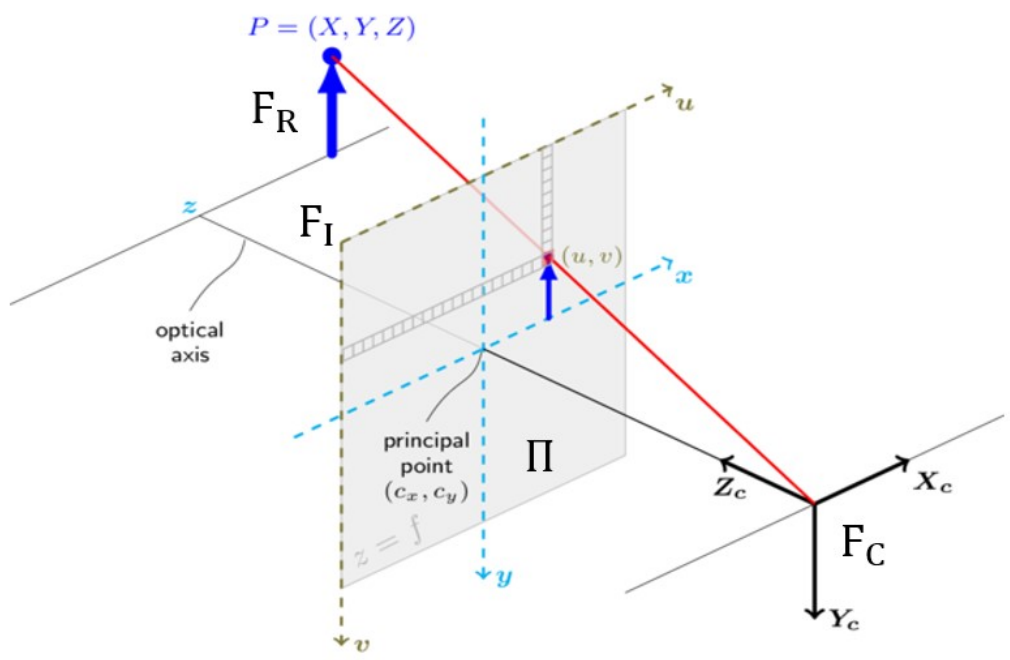

Fig. 4. Sketch of the perspective projection model reference frames (from [14]), highlighting the real world coordinate frame $\mathrm{F}_{\mathrm{R}}$, the IR camera frame $\mathrm{F}_{\mathrm{C}}$ and the image frame $\mathrm{F}_{\mathrm{I}}$.

The optical calibration here adopted exploits the pinhole camera calibration model available in the Open Source Computer Vision Library (OpenCV) [14], which is based on a perspective projection with lens distortion proposed by Heikkila [9]. Let's first consider a model of pure perspective projection, as depicted in Fig. 4 . The centre of projection is the origin of the camera frame $\mathrm{F}_{\mathrm{C}}$. The image plane $\Pi$ is parallel to the $X_{C}-Y_{C}$ plane, at a distance $f$ (focal length) from the projection centre, along the $z$ axis. The $z$ axis is also called optical axis, and its intersection with the plane $\Pi$ is called principal point. The axes $u$ and $v$ of the 2D image frame $F_{I}$ are parallel to the $x$ and $y$ axes and the coordinates of the principal point are $\left[c_{x}, c_{y}\right]$. The coordinates of a point $P$ in the real world frame $F_{R}$ are $[X, Y, Z]$, while in the camera frame they are $[x, y, z]$ and in the camera frame they are $[u, v]$.

The transformation between the real world coordinates and the camera frame coordinates can be formulated as

$$
\left[\begin{array}{l}
x \\
y \\
Z
\end{array}\right]=[\boldsymbol{R} \mid \boldsymbol{t}]\left[\begin{array}{c}
X \\
Y \\
Z \\
1
\end{array}\right],
$$

through the rotation matrix $R$ and the translation vector $\boldsymbol{t}$

$$
[\boldsymbol{R} \mid \boldsymbol{t}]=\left[\begin{array}{llll}
R_{11} & R_{12} & R_{13} & t_{1} \\
R_{21} & R_{22} & R_{23} & t_{2} \\
R_{31} & R_{32} & R_{33} & t_{3}
\end{array}\right],
$$

while the transformation between the camera frame coordinates and the image frame coordinates is

$$
\left[\begin{array}{l}
u \\
v \\
1
\end{array}\right]=A\left[\begin{array}{l}
x \\
y \\
z
\end{array}\right]
$$

where $A$ is the perspective transformation matrix

$$
A=\left[\begin{array}{ccc}
f_{x} & 0 & c_{x} \\
0 & f_{y} & c_{y} \\
0 & 0 & 1
\end{array}\right]
$$

$\boldsymbol{R}$ is an orthonormal matrix, which can be described by three Euler angles $\varphi, \theta$ and $\psi$. The three translation components and three rotation angles are called extrinsic parameters of the projection, while $f_{x}, f_{y}, c_{x}$ and $c_{y}$ are called intrinsic projection parameters.

However, a pure perspective projection is typically not sufficient to model real cameras accurately. Lenses, in fact, can introduce significant distortion in the optical path of the light rays. The distorted coordinates in the image frame can be obtained by applying a distortion model, which is usually decomposed into its radial and tangential components. The optical calibration model uses three radial distortion coefficients, $k_{1}, k_{2}$ and $k_{3}$, and two tangential distortion coefficients, $p_{1}$ and $p_{2}$, and the distorted coordinates in the camera frame can be represented by the following additional transformation

$$
\left[\begin{array}{l}
x_{d} \\
y_{d}
\end{array}\right]=\left[\begin{array}{l}
x^{\prime}\left(1+k_{1} r^{2}+k_{2} r^{4}+k_{3} r^{6}\right)+2 p_{1} x^{\prime} y^{\prime}+p_{2}\left(r^{2}+2 x^{\prime 2}\right) \\
y^{\prime}\left(1+k_{1} r^{2}+k_{2} r^{4}+k_{3} r^{6}\right)+p_{1}\left(r^{2}+2 y^{\prime 2}\right)+2 p_{2} x^{\prime} y^{\prime}
\end{array}\right]
$$

where $x^{\prime}=x / z, y^{\prime}=y / z, r^{2}=x^{\prime 2}+y^{\prime 2}$ and the subscript $\mathrm{d}$ indicates the distorted coordinates. 


\subsubsection{Optical calibration procedure}

A dedicated geometrical calibration procedure is necessary to determine the extrinsic and intrinsic projection parameters, along with the distortion coefficients. This is generally accomplished with the help of a calibration target. This object must be provided with detectable points, in order for them to be recognised in the thermal image. Two different techniques are reported in the literature: either the calibration points are embedded in the object of interest (in situ calibration), as it was proposed by Le Sant et al. [5, 6, 7], or an external target, typically a planar surface, is exploited, as accurately described by Cardone et al. [8]. In the former case, only a limited number of calibration spots can be placed on the test object, while in the latter case a pattern of equidistant points in two orthogonal directions is arranged on a calibration plate.

In the context of this work, however, owing to the difficulties arising in mounting and precisely orienting a calibration plate in the Plasmatron facility, an ad hoc calibration cap, shown in Fig. 5 (left), has been designed and manufactured. The cap is made of aluminium and features $411 \mathrm{~mm}$ diameter calibration holes, drilled at known positions. The desired contrast, which allows the calibration spots to be detected in the thermal image, is achieved exploiting different emissivity values. Aluminium, in fact, is characterized by a low emissivity coefficient and the calibration spots become radiating cavities, which are then characterized by large values of emissivity and can be easily detected in the thermal image. This cap is placed on the test sample before the test to acquire a calibration thermogram (Fig. 5 (right)) and removed for the test. Moreover, in order to increase their contrast, the probe can be additionally heated by means of a heat gun before mounting the cap.
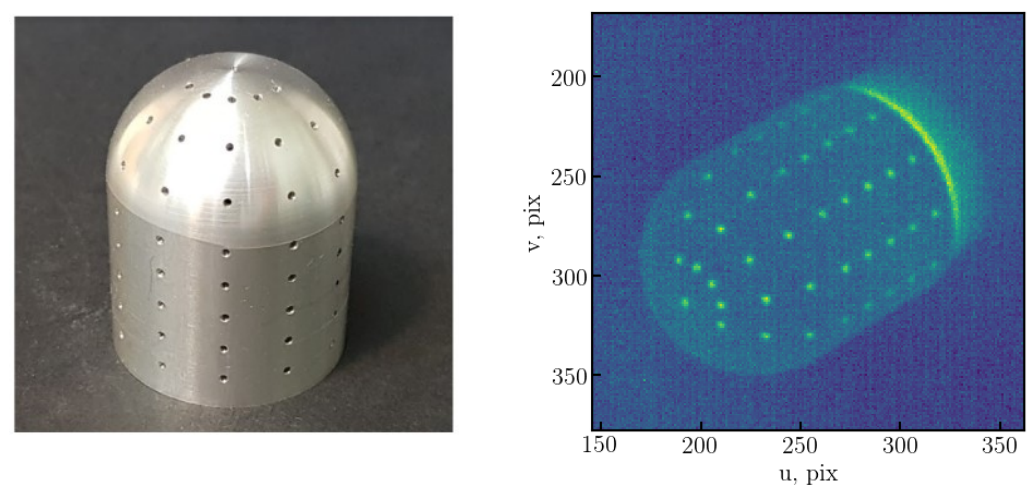

Fig. 5. Picture of the aluminium calibration cap (left) and corresponding IR thermogram (right) with detectable spots.

Once a reference coordinate system has been set, the real world coordinates of the calibration spots are known. A calibration thermogram is then acquired and the position of the calibration points in the image frame are automatically detected by means of suitable image processing techniques. Hence, one can apply the camera calibration algorithm: the extrinsic and intrinsic parameters, along with the distortion constants, can be found with a minimization of the root mean square difference between the known image coordinates of the control points and their image coordinates computed in accordance with the optical projection model. The typical reprojection error associated to the calibration procedure is \pm 0.5 pixel.

\subsubsection{Time-resolved surface recession and sample shape measurements}

As already introduced in section 1, as the graphite sample is exposed to the plasma flow, heterogeneous chemical reactions between the sample surface and the plasma flow result in recession and shape change of the test material. Hence, the initial test object geometry evolves in time in a hardly predictable manner. While the calibration parameters found with the optical calibration procedure are still valid, the object shape is no longer the initial one and the coordinates of the test object surface points in the real world frame $F_{R}$ become unknown. To this purpose, we propose to exploit an additional measurement to infer the object shape. The Phantom 7.1 CMOS high-speed camera (Vision Research, Wayne, New Jersey, USA), featuring a $200 \mathrm{~mm}$ focal length lens, is adopted for the in-situ recession analysis of the ablating test sample. A calibration chessboard, placed at the initial sample location, provides the necessary pixel to length calibration and an acquisition rate of $10 \mathrm{~Hz}$ is used.

Edge detection algorithms, available in the OpenCV library are then applied to the recorded frames. Once the edge pixels are located, the measured shape is fitted with a combination of elliptical and linear shape functions. Finally, under the assumption of axisymmetry, the three-dimensional object shape can be easily inferred from the observed twodimensional projection. This procedure allows to retrieve the position of the sample surface points in time as $[X(t), Y(t), Z(t)]$. Exploiting the precise synchronization between the IR camera and the high-speed camera, the timedependent test object shape can be projected onto the IR camera image frame, by means of the previously described optical calibration model, allowing three-dimensional temperature maps reconstructions on the ablating surface. 


\section{Results}

The proposed procedure has been applied to analyse the ablation of the $30 \mathrm{~mm}$ hemisphere-cylinder graphite sample. The test was performed in air plasma, allowing $\sim 840 \mathrm{~s}$ exposure to the flow. With reference to section 2.1., the Plasmatron operating conditions are reported in table 1.

Table 1. Plasmatron operating conditions.

\begin{tabular}{ccccc}
\hline$p_{\mathrm{s}}$ & $\dot{m}_{\text {gas }}$ & $\dot{q}_{\mathrm{cw}}$ & $p_{\text {dyn }}$ & $P_{\text {el }}$ \\
$\mathrm{mbar}$ & $\mathrm{g} / \mathrm{s}$ & $\mathrm{kW} / \mathrm{m} 2$ & $\mathrm{~Pa}$ & $\mathrm{~kW}$ \\
\hline $100 \pm 0.2$ & 16 & $916 \pm 91$ & $7 \pm 3$ & 151.9 \\
\hline
\end{tabular}

The measured surface temperature in the stagnation region of the test sample by means of the two-colour pyrometer, as well as the correspondent IR camera temperature before in-band emissivity correction (hence using $\varepsilon_{\Delta \lambda}=1$ ) are reported in Fig 6 (left). $t=0 \mathrm{~s}$ corresponds to the probe injection in the plasma flow. One can notice that the IR camera measurement, corrected for $\varepsilon_{\Delta \lambda}=1$, results in a lower temperature with respect to the one measured by the emissivity-independent two colour pyrometer. This confirms that $\varepsilon_{\Delta \lambda}$ should be lower than one, as expected. Applying the procedure for the in-band emissivity measurement, proposed in section 2.2.5., the temperature dependence of $\varepsilon_{\Delta \lambda}$ can be retrieved, as shown in Fig. 6 (right). The value of $\varepsilon_{\Delta \lambda}$ is in very good agreement with typical values of spectral emissivity of graphite [15], which corroborates the proposed measurement procedure. The measured values of $\varepsilon_{\Delta \lambda}$ are then fitted with a third order polynomial function, and used to correct each IR camera frame of the video sequence.
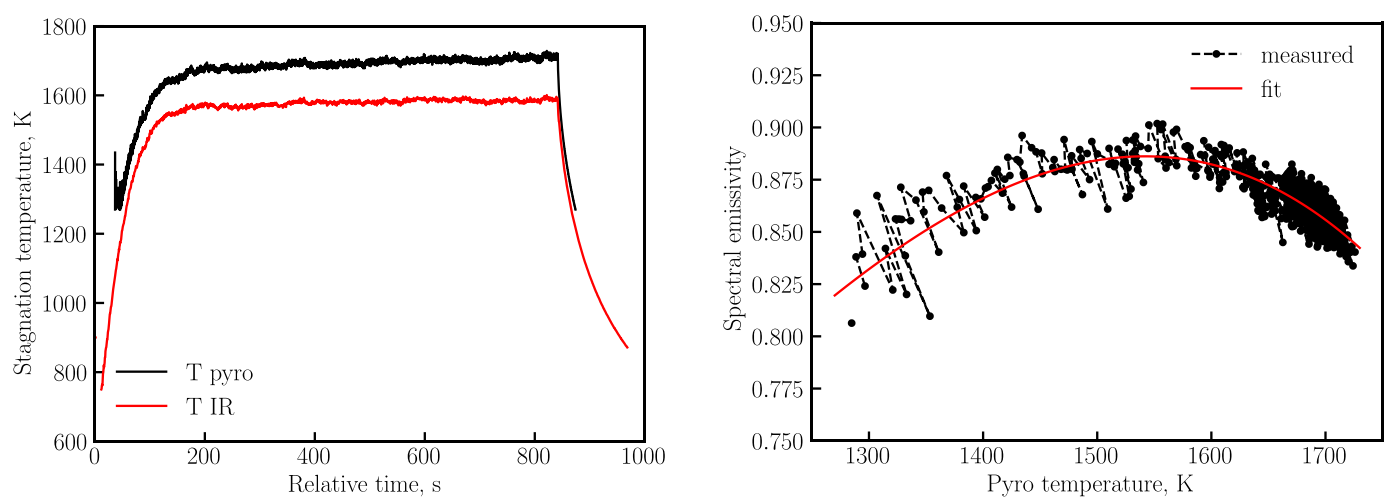

Fig. 6. (left) Time-resolved stagnation region temperature measurement by means of two-color pyrometry (black line) and IR thermography (red line), using an in-band emissivity coefficient equal to 1 for the latter. (right) Measured temperature evolution of the in-band emissivity coefficient (black curve) and its third order polynomial fit (red curve).
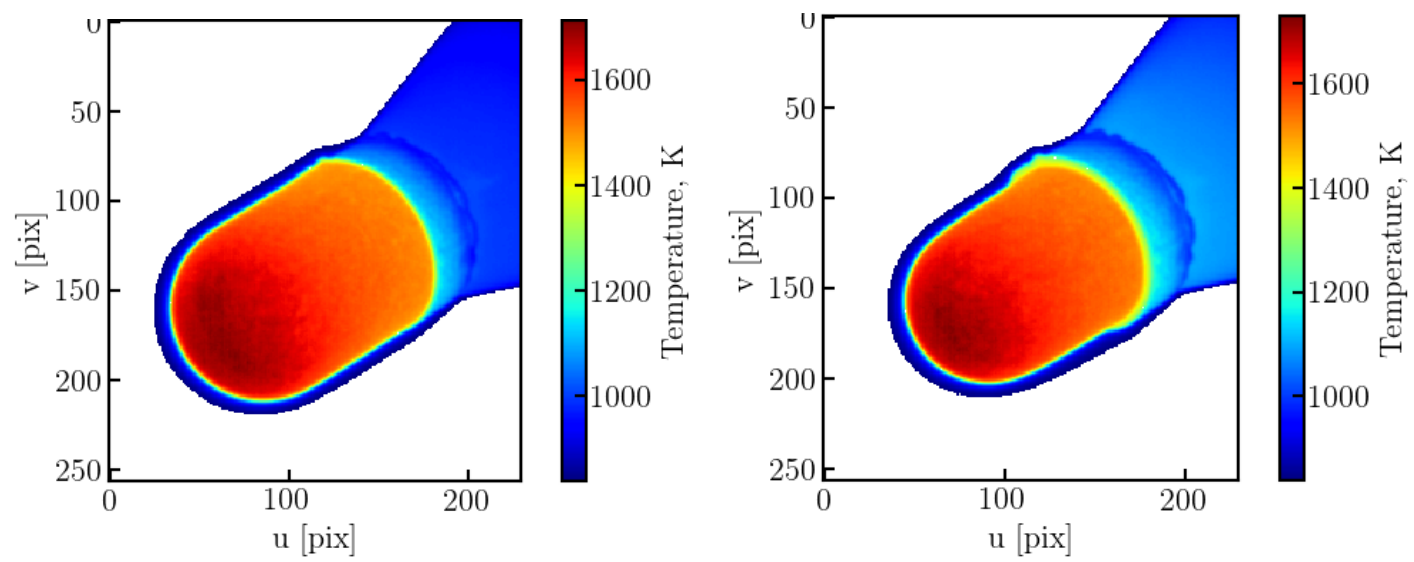

Fig. 7. Example of IR camera frames corrected for temperature-dependent in-band emissivity and calibrated in temperature for two time instants of the test: $t=200.26 \mathrm{~s}$ (left) and $t=841.26 \mathrm{~s}$ (right), highlighting how the sample shape changed during the test time. 
Two representative IR camera frames (at $t=200.26 \mathrm{~s}$ and $t=841.26 \mathrm{~s}$ ), corrected for in-band emissivity and calibrated in temperature, are shown in Fig. 7. We can notice how, as solid carbon is removed from the sample surface by heterogeneous chemical reactions with the plasma flow, the shape of the test sample changes in time. Fig. 8 shows an example of the recorded HSC frame at the end of the test time $(t=841.26 \mathrm{~s})$ and the detected test sample shape retrieved by means of edge detection algorithms. By applying this procedure to each frame, the time evolution of the sample shape can be obtained (Fig. 8 (right)). Once this information is available, one can reconstruct the three-dimensional shape of the test sample under the assumption of axisymmetry. After the time history of the IR camera and HSC measurements are synchronized, the reconstructed sample shape is used for the three-dimensional temperature map reconstruction on the ablating surface, as represented in Fig. 9.
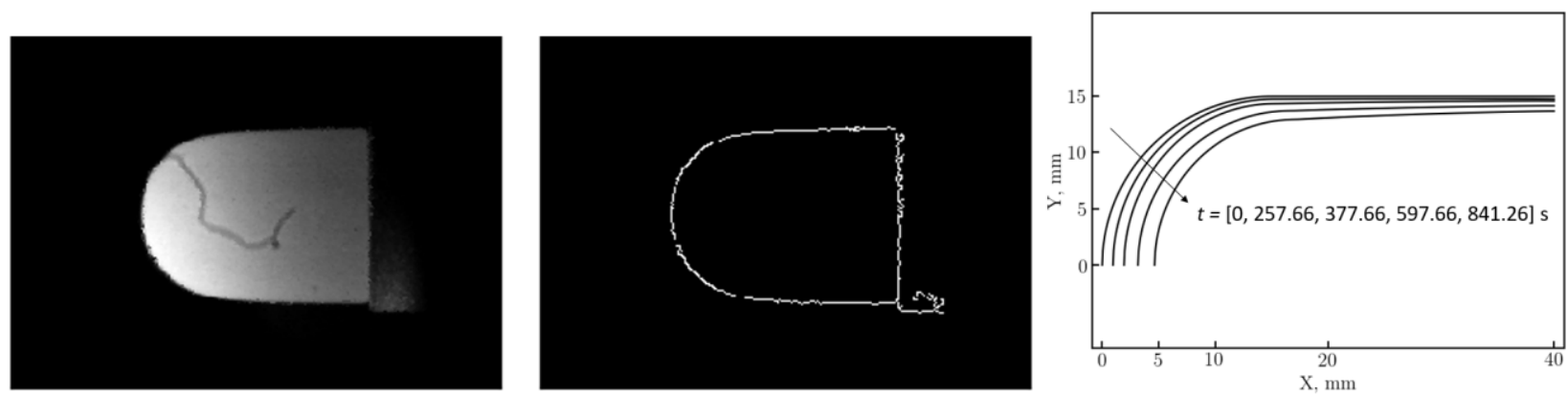

Fig. 8. Example of high-speed camera frame (left) and detected edge shape (middle) at $t=841.26 \mathrm{~s}$, corresponding to the end of the test time. (right) Fitted shapes of the test sample material, starting from the edge detection applied to the HSC frames, at different times during the test. Notice the significant ablation around the stagnation region ( $5 \mathrm{~mm})$ as well as in the lateral region ( 1.2 $\mathrm{mm}$ ), which result in a considerable shape change.
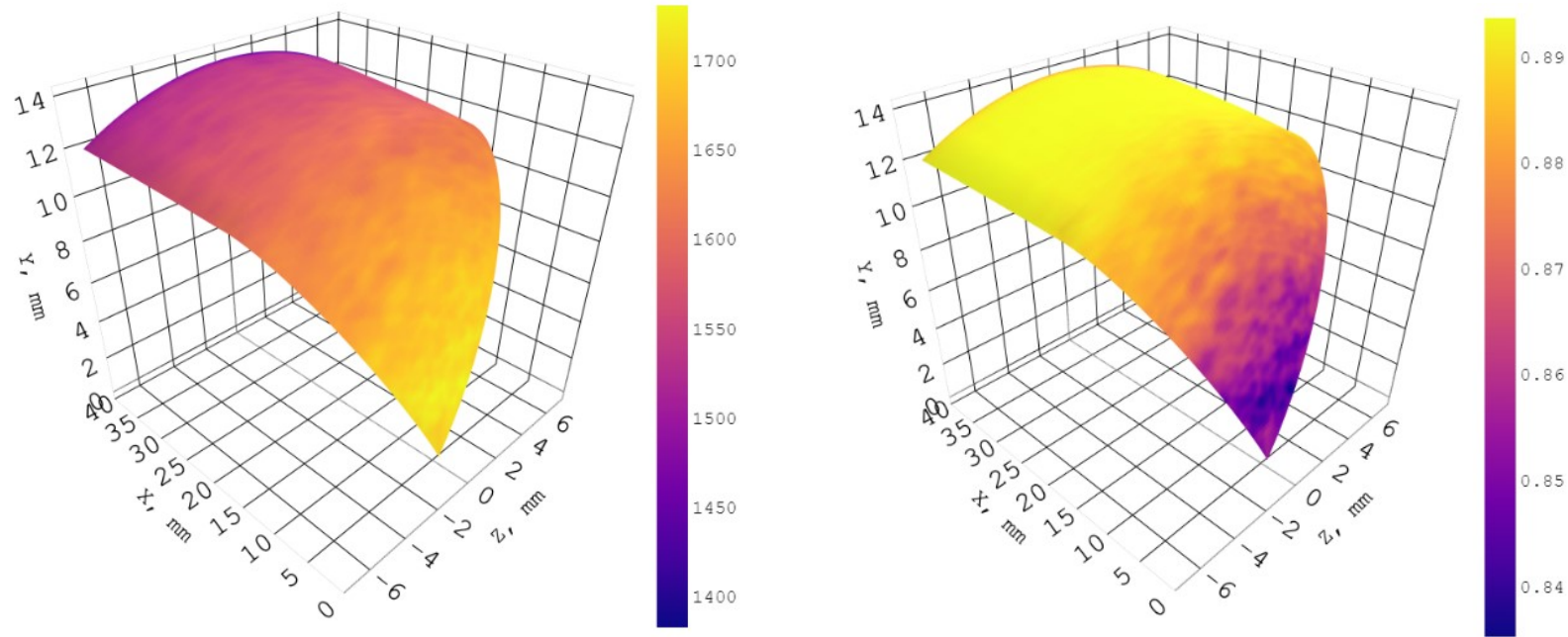

Fig. 9. Example of $3 D$ reconstruction of the temperature map (left) (temperature units in Kelvin) and in-band emissivity map (right) on the test article shape at $t=841.26 \mathrm{~s}$, corresponding to the end of the test time. Notice the ablated sample geometry.

\section{Conclusions}

In this work a novel technique, which allows to reconstruct three-dimensional temperature maps over an ablating sample exposed to a plasma flow, has been shown. An optical geometric calibration, featuring a perspective projection with lens distortion, is first applied to IR thermography in order to retrieve the necessary mapping between the real world coordinate frame and the IR camera image frame. The root mean square error of the mapping is about 0.5 pixels. Highspeed camera imaging and edge detection algorithms are then used to record the time evolution of the test object shape and infer its three-dimensional geometry under the assumption of axisymmetry. Specific procedures for the in situ measurement of the in-band emissivity and its temperature dependent correction allow precise evaluation of the object surface temperature. The proposed technique was successfully applied to reconstruct three-dimensional temperature 
maps over an ablating graphite sample exposed to a plasma flow in the VKI Plasmatron facility. Experimental results show a significant surface recession of the test sample around the stagnation region $(\sim 5 \mathrm{~mm})$ as well as in the lateral region $(\sim 1.2 \mathrm{~mm})$ during the $\sim 840 \mathrm{~s}$ test time, contributing to a relevant shape change. The measured time evolution of the test sample shape is fed to the optical calibration algorithm, which allows its projection onto the IR camera image frame. 3D temperature maps, as well as emissivity maps are finally reconstructed in a time varying fashion.

The proposed methodology, featuring in-situ measurement of the in-band emissivity and three-dimensional temperature maps reconstruction on a dynamic target, can allow significant improvements in the material response characterization techniques in plasma wind tunnel testing. Precise spatial accuracy in the temperature measurements, in fact, is needed to provide valuable information for comparison and validation of numerical simulation tools for the material thermal response.

As a feature work, a careful investigation on the axisymmetry assumption in the reconstruction of the time-varying object shape must be performed. Moreover, dedicated uncertainty analysis, propagating the error retrieved from the highspeed camera shape through the optical calibration model for the IR camera can provide a deeper insight into the overall spatial accuracy of the technique. Further investigation on the angular dependence of the in-band emissivity and its accurate correction depending on the computed viewing angle from the 3D reconstruction would also allow further improvement in the temperature measurement accuracy.

\section{Aknowledgements}

The research of A. Fagnani is supported by a fellowship awarded by the Research Foundation Flanders (FWO \#1SB3219N). Pascal Collin is aknowledged for his valuable help as Plasmatron operator.

\section{REFERENCES}

[1] Chazot, O., Régnier, R., and Garcia, M. A., "Simulation Methodology in Plasmatron Facility and Hypersonic Wind Tunnels," 12th International Conference on Methods of Aerophysical Research, 2004, pp. 87-92.

[2] Panerai, F. Aerothermochemistry Characterization of Thermal Protection Systems. Ph.D. Thesis, von Karman Institute for Fluid Dnamics \& Università degli Studi di Perugia, 2012.

[3] Helber, B., Turchi, A., Scoggins, J. B., Hubin, A., and Magin, T. E. "Experimental Investigation of Ablation and Pyrolysis Processes of Carbon-Phenolic Ablators in Atmospheric Entry Plasmas." International Journal of Heat and Mass Transfer, Vol. 100, 2016, pp. 810-824.

[4] Prévereaud, Y., Vérant, J. L., Balat-Pichelin, M., and Moschetta, J. M. "Numerical and Experimental Study of the Thermal Degradation Process during the Atmospheric Re-Entry of a TiAl6V4 Tank." Acta Astronautica, Vol. 122, 2016, pp. 258-286.

[5] Le Sant Y., Marchand M., Millanb P., Fontaine J. - An overview of infrared thermography techniques used in large wind tunnels - Aerospace Science and Technology, Vol.6, pp. 355-366, 2002;

[6] Le Sant Y., Deleglise B., Mebarki Y. - An automatic image alignment method applied to pressure sensitive paint measurements - 17th ICl-ASF, Monterey, USA, 1997;

[7] Le Sant Y., Mérienne M-C. - An image resection method applied to mapping techniques - 16th ICIASF, WPAFB, Ohio, USA, 1995;

[8] Cardone G., laniro A., dello lonio G., Passaro A. - Temperature maps measurements on 3D surfaces with infrared thermography - Experiments in Fluids, Vol. 52, pp. 375-285, 2012.

[9] Heikkilä J., Silven O. - Geometric camera calibration using circular control points - IEEE Trans Pattern Anal Mach Intell, Vol. 22(10), pp. 1077-1077;

[10] G. M. Carlomagno and G. Cardone. Infrared thermography for convective heat transer measurements. Experiments in Fluids, 49:1187-1218, 2010

[11] Gülhan A. - Applicatiton of pyrometry and IRthermography to high surface temperature measurements Defense technical information center notice ADP010751;

[12] Degrez, G., Vanden Abeele, D., Barbante, P., and Bottin, B., "Numerical Simulation of Inductively Coupled Plasma Flows under Chemical Non-equilibrium," International Journal of Numerical Methods for Heat \& Fluid Flow, Vol. 14, No. 4, 2004,pp. 538-558.

[13] Vanden Abeele, D., and Degrez, G., "Efficient computational model for inductive plasmas flows," AIAA J., Vol. 38, No. 2, 2000, pp. 234-242.

[14] OpenCV (Open Source Computer Vision Library), available at https://opencv.org

[15] Neuer, G. "Spectral and Total Emissivity Measurements of Highly Emitting Materials." International Journal of Thermophysics, Vol. 16, No. 1, 1995, pp. 257-265. 